\title{
Stability Conditions for Fuzzy Control Systems with Fuzzy Feedback Gains
}

\author{
H.K. Lam, Member, IEEE, C.W. Yeung, and F.H.F. Leung, Senior Member, IEEE
}

\begin{abstract}
This paper presents the stability analysis of fuzzy-model-based control systems. A fuzzy controller with fuzzy feedback gains is proposed to control a nonlinear system represented by the T-S fuzzy model. The fuzzy feedback gains effectively enhance the nonlinearity of the fuzzy controller to strengthen the stabilization ability. To facilitate the stability analysis and reduce the computational demand, the fuzzy controller is separated from the fuzzy model by a particular system representation during analysis. Consequently, the number of stability conditions can be reduced to ease the finding of the solution. Two cases of fuzzy controller design, namely the fuzzy controller employs the same and different membership functions as those of the fuzzy plant model, are considered for various applications. Simulation examples are given to illustrate the merits of the proposed approach.
\end{abstract}

\section{INTRODUCTION}

Fuzzy-model-based control approach is a promising approach to facilitate the system stability analysis and the design of the fuzzy controller based on the T-S fuzzy model [1]-[2]. Flourishing results [3]-[12] were reported on guaranteeing the system stability of fuzzy-model-based control systems. The stability conditions can be expressed in terms of LMIs (linear matrix inequalities) of which the solution can be solved efficiently and numerically using some convex programming techniques.

To investigate the system stability of the fuzzy-model-based control systems, a T-S fuzzy model is employed to represent the nonlinear plant. A fuzzy controller [3]-[12] is proposed to close the feedback loop. The resulting fuzzy-model-based control system can be represented as a weighted average sum of linear sub-control systems. In [3]-[4], basic stability conditions were derived based on the Lyapunov's stability theory. The membership functions of the fuzzy controller in [3]-[4] can be designed arbitrarily; hence, it offers design flexibility to the controllers. Simple membership functions can be employed to lower the structural complexity and implementation cost of the fuzzy controller. Furthermore, as the membership functions of the fuzzy plant model do not contribute to the stability conditions, the fuzzy controller exhibits inherent robustness property to nonlinear

This work described in this paper was partially supported by the King's College London and a grant from the Research Grants Council of the Hong Kong Special Administrative Region, China (Project No. PolyU 5218/06E).

H.K. Lam is with the Division of Engineering, The King's College London, Strand, London, WC2R 2LS, United Kingdom (e-mail: hak-keung.lam@kcl.ac.uk).

C.W. Yueng is with Centre for Signal Processing, Department of Electronic and Information Engineering, The Hong Kong Polytechnic University, Hung Hom, Kowloon, Hong Kong (e-mail: encwy@eieserver.eie.polyu.edu.hk).

F.H.F. Leung is with the Centre for Signal Processing, Department of Electronic and Information Engineering, The Hong Kong Polytechnic University, Hung Hom, Kowloon, Hong Kong (e-mail: enfrank@inet.polyu.edu.hk). plants subject to parameter uncertainties, which are embedded in the membership functions of the fuzzy plant model. However, the derived stability conditions are often very conservative [3]-[4].

To relax the conservativeness of the stability conditions, fuzzy controllers that share the same premise membership functions with the fuzzy plant model were proposed [4]-[5]. Under such a design criterion, the design flexibility of fuzzy controller is lowered. Moreover, the complicated membership functions will increase the structural complexity and implementation cost of the fuzzy controller. Also, the membership functions of the fuzzy plant model must be known, meaning that the nonlinear plant must be uncertainty free. Consequently, the inherent robustness property of the fuzzy controller in [3]-[4] vanishes. Further relaxed stability conditions can be found in [4]-[12].

In this paper, a fuzzy controller with fuzzy feedback gains is proposed for nonlinear plants. It was shown that the fuzzy controller in [14]-[15] with variable feedback gains can further strengthen its stabilization ability thanks to the enhanced nonlinearity to compensate for the unstable elements of the nonlinear plants. However, the fuzzy feedback gains, which increase the order of the summation terms, complicate the stability analysis and lead to a large number of stability conditions. Moreover, when the stability analysis approach in [6]-[12] is employed, a large matrix governing the system stability will be formed. Hence, the computational demand for finding the solution of the stability conditions will be increased seriously. The situation will be worsened especially when the number of rules of the fuzzy plant model is large. In this paper, the proposed fuzzy controller is separated from the fuzzy plant model during the system representation for doing analysis to reduce the number of stability conditions and computational complexity. Two cases of fuzzy controllers, which employ the same and different membership functions as those of the fuzzy plant model, are investigated.

\section{FUZZY MODEL AND FUZZY CONTROLLER}

A fuzzy-model-based control system comprising a nonlinear plant represented by a fuzzy model and a fuzzy controller connected in closed loop is considered.

\section{A. Fuzzy Model}

Let $p$ be the number of fuzzy rules describing the behaviour of the nonlinear plant. The $i$-th rule is of the following format: Rule $i$ : IF $f_{1}(\mathbf{x}(t))$ is $\mathrm{M}_{1}^{i}$ AND ... AND $f_{\Psi}(\mathbf{x}(t))$ is $\mathbf{M}_{\Psi^{\prime}}^{i}$

$$
\text { THEN } \dot{\mathbf{x}}(t)=\mathbf{A}_{i} \mathbf{x}(t)+\mathbf{B}_{i} \mathbf{u}(t)
$$


where $\mathbf{M}_{\alpha}^{i}$ is a fuzzy term of rule $i$ corresponding to the function $f_{\alpha}(\mathbf{x}(t)), \alpha=1,2, \ldots, \Psi ; i=1,2, \ldots, p ; \Psi$ is a positive integer; $\mathbf{A}_{i} \in \mathfrak{R}^{n \times n}$ and $\mathbf{B}_{i} \in \mathfrak{R}^{\mathfrak{r} \times m}$ are known constant system and input matrices respectively; $\mathbf{x}(t) \in \mathfrak{R}^{r \ltimes 1}$ is the system state vector and $\mathbf{u}(t) \in \mathfrak{R}^{m \times 1}$ is the input vector. The system dynamics is described by,

$$
\begin{aligned}
& \dot{\mathbf{x}}(t)=\sum_{i=1}^{p} w_{i}(\mathbf{x}(t))\left(\mathbf{A}_{i} \mathbf{x}(t)+\mathbf{B}_{i} \mathbf{u}(t)\right) \\
& \sum_{i=1}^{p} w_{i}(\mathbf{x}(t))=1, w_{i}(\mathbf{x}(t)) \in\left[\begin{array}{ll}
0 & 1
\end{array}\right] \text { for all } i \\
& w_{i}(\mathbf{x}(t))=\frac{\mu_{M_{1}^{\prime}}\left(f_{1}(\mathbf{x}(t))\right) \times \mu_{M_{2}^{\prime}}\left(f_{2}(\mathbf{x}(t))\right) \times \cdots \times \mu_{M_{\Psi}^{\prime}}\left(f_{\Psi}(\mathbf{x}(t))\right)}{\sum_{k=1}^{p}\left(\mu_{M_{1}^{k}}\left(f_{1}(\mathbf{x}(t))\right) \times \mu_{M_{2}^{k}}\left(f_{2}(\mathbf{x}(t))\right) \times \cdots \times \mu_{M_{\Psi}^{k}}\left(f_{\Psi}(\mathbf{x}(t))\right)\right.}
\end{aligned}
$$

is a nonlinear function of $\mathbf{x}(t)$ and $\mu_{\mathrm{M}_{\alpha}^{i}}\left(f_{\alpha}(\mathbf{x}(t))\right), \alpha=1,2, \ldots$, $\Psi$, is the grade of membership corresponding to the fuzzy term $\mathbf{M}_{\alpha}^{i}$. It should be noted that $\mu_{\mathrm{M}_{\alpha}^{i}}\left(f_{\alpha}(\mathbf{x}(t))\right)$ are uncertain when the nonlinear plant is subject to parameter uncertainties.

\section{B. Fuzzy Controller}

A fuzzy controller with $p$ rules is considered. The $j$-th rule of the fuzzy controller is defined as follows.

Rule $j$ : IF $g_{1}(\mathbf{x}(t))$ is $\mathrm{N}_{1}^{j} \mathrm{AND} \ldots$ AND $g_{\Omega}(\mathbf{x}(t))$ is $\mathrm{N}_{\Omega}^{j}$

$$
\text { THEN } \mathbf{u}(t)=\mathbf{F}_{j} \mathbf{G}(\mathbf{x}(t)) \mathbf{x}(t), j=1,2, \ldots, p
$$

where $\mathbf{N}_{\beta}^{j}$ is a fuzzy term of rule $j$ corresponding to the function $g_{\beta}(\mathbf{x}(t)), \beta=1,2, \ldots, \Omega, j=1,2, \ldots, p ; \Omega$ is a positive integer; $\mathbf{F}_{j} \in \mathfrak{R}^{m \times n}$ and $\mathbf{G}(\mathbf{x}(t)) \in \mathfrak{R}^{r \times n}$ are the constant and nonlinear feedback gains of rule $j$ respectively. The inferred output of the fuzzy controller is given by,

$$
\begin{aligned}
& \mathbf{u}(t)=\sum_{j=1}^{p} m_{j}(\mathbf{x}(t)) \mathbf{F}_{j} \mathbf{G}(\mathbf{x}(t)) \mathbf{x}(t) \\
& \sum_{j=1}^{p} m_{j}(\mathbf{x}(t))=1, m_{j}(\mathbf{x}(t)) \in\left[\begin{array}{ll}
0 & 1
\end{array}\right] \text { for all } j \\
& m_{j}(\mathbf{x}(t))=\frac{\mu_{\mathrm{Nj}}\left(g_{1}(\mathbf{x}(t))\right) \times \mu_{\mathrm{Ni}}\left(g_{2}(\mathbf{x}(t))\right) \times \cdots \times \mu_{\mathrm{N}_{\Omega}^{j}}\left(g_{\Omega}(\mathbf{x}(t))\right)}{\sum_{k=1}^{p}\left(\mu_{\mathrm{N}_{1}^{(}}\left(g_{1}(\mathbf{x}(t))\right) \times \mu_{\mathrm{N}_{2}^{v_{2}}}\left(g_{2}(\mathbf{x}(t))\right) \times \cdots \times \mu_{\mathrm{N}_{\Omega}^{k}}\left(g_{\Omega}(\mathbf{x}(t))\right)\right)}
\end{aligned}
$$

is a nonlinear function of $\mathbf{x}(t)$ and $\mu_{N_{\beta}^{j}}\left(g_{\beta}(\mathbf{x}(t))\right)$ is the grade of membership corresponding to the fuzzy term $\mathrm{N}_{\beta}^{j}$.

The feedback gains of $\mathbf{G}(\mathbf{x}(t))$ are governed by the following rules.

Rule $k$ : IF $g_{1}(\mathbf{x}(t))$ is $\mathrm{N}_{1}^{k}$ AND ... AND $g_{\Omega}(\mathbf{x}(t))$ is $\mathbf{N}_{\Omega}^{k}$

$$
\text { THEN } \mathbf{G}(\mathbf{x}(t))=\mathbf{G}_{k}, k=1,2, \ldots, p
$$

The inferred feedback gain is defined as,

$$
\mathbf{G}(\mathbf{x}(t))=\sum_{k=1}^{p} m_{k}(\mathbf{x}(t)) \mathbf{G}_{k}
$$

where $\mathbf{G}_{k} \in \Re^{n \times n}, k=1,2, \ldots, p$, are constant feedback gains to be designed. From (6) to (10), we have

$$
\mathbf{u}(t)=\sum_{j=1}^{p} \sum_{k=1}^{p} m_{j}(\mathbf{x}(t)) m_{k}(\mathbf{x}(t)) \mathbf{F}_{j} \mathbf{G}_{k} \mathbf{x}(t)
$$

Remark 1: It should be noted that the fuzzy controller of (11) is reduced to the traditional one in [3]-[12] when $\mathbf{G}_{k}=\mathbf{I}$ for all $k$, where $\mathbf{I}$ denotes the identity matrix of appropriate dimension. When $\mathbf{G}_{k} \neq \mathbf{I}$, the fuzzy feedback gain of $\mathbf{G}(\mathbf{x}(t))$ effectively enhances the nonlinearity of the fuzzy controller to compensate for the unstable elements of the nonlinear plant. In other words, the additional feedback gains of $\mathbf{G}_{k}$ increase the degree of freedom on searching for the solution of the stability conditions. Consequently, the stabilization ability of the proposed fuzzy controller is strengthened.

\section{Stablity ANALYSis}

In this section, the system stability of the fuzzy-model-based control systems formed by the nonlinear plant of (2) and the fuzzy controller of (11) connected in a closed loop is investigated. From (2) and (11), with the property of $\sum_{i=1}^{p} w_{i}(\mathbf{x}(t))=\sum_{j=1}^{p} m_{j}(\mathbf{x}(t))=$ $\sum_{i=1}^{p} \sum_{j=1}^{p} \sum_{k=1}^{p} w_{i}(\mathbf{x}(t)) m_{j}(\mathbf{x}(t)) m_{k}(\mathbf{x}(t))=1$, we have,

$$
\begin{gathered}
\dot{\mathbf{x}}(t)=\sum_{i=1}^{p} w_{i}(\mathbf{x}(t))\left(\mathbf{A}_{i} \mathbf{x}(t)+\mathbf{B}_{i} \sum_{j=1}^{p} \sum_{k=1}^{p} m_{j}(\mathbf{x}(t)) m_{k}(\mathbf{x}(t)) \mathbf{F}_{j} \mathbf{G}_{k} \mathbf{x}(t)\right) \\
=\sum_{i=1}^{p} \sum_{j=1}^{p} \sum_{k=1}^{p} w_{i}(\mathbf{x}(t)) m_{j}(\mathbf{x}(t)) m_{k}(\mathbf{x}(t))\left(\mathbf{A}_{i}+\mathbf{B}_{i} \mathbf{F}_{j} \mathbf{G}_{k}\right) \mathbf{x}(t)
\end{gathered}
$$

It can be seen from (12) that the fuzzy-model-based control system consists of three summation terms. By using the analysis approach in [3]-[12], the number of stability conditions will increase with the number of summation terms. Moreover, in [5]-[12], the stability condition will be formulated in a larger matrix which further increases the computational demand to find the solution of the stability conditions. In some cases, a numerical solution cannot be found owing to the limited memory of the computer even though a feasible solution actually exists, especially when the number of rules of the fuzzy model is large.

For brevity, $w_{i}(\mathbf{x}(t)), m_{j}(\mathbf{x}(t))$ and $m_{k}(\mathbf{x}(t))$ are written as $w_{i}$, $m_{j}$ and $m_{k}$ respectively. From (11), the fuzzy controller can be expressed as follows.

$$
\begin{aligned}
\mathbf{u}(t) & =\sum_{j=1}^{p} m_{j} \mathbf{F}_{j} \mathbf{y}(t) \\
\mathbf{y}(t) & =\sum_{j=1}^{p} m_{j} \mathbf{G}_{j} \mathbf{x}(t)
\end{aligned}
$$

From (2), (13) and (14), we have the following property to facilitate the stability analysis.

$$
\sum_{j=1}^{p} m_{j}\left[\begin{array}{ccc}
\mathbf{0} & \mathbf{0} & \mathbf{0} \\
\mathbf{G}_{j} & -\mathbf{I} & \mathbf{0} \\
\mathbf{0} & \mathbf{F}_{j} & -\mathbf{I}
\end{array}\right]\left[\begin{array}{l}
\mathbf{x}(t) \\
\mathbf{y}(t) \\
\mathbf{u}(t)
\end{array}\right]=\left[\begin{array}{l}
\mathbf{0} \\
\mathbf{0} \\
\mathbf{0}
\end{array}\right]
$$

The system stability of the fuzzy-model-based control system of (12) is investigated using the following Lyapunov function candidate.

$V(t)=\mathbf{x}(t)^{\mathrm{T}} \mathbf{P}_{1} \mathbf{x}(t)$

where $\mathbf{P}_{1}=\mathbf{P}_{1}^{\mathrm{T}} \in \mathfrak{R}^{\wedge \ltimes n}>0$. Stability conditions are derived to ensure that $\dot{V}(t)<0$, which implies the asymptotic stability of 
the fuzzy-model-based control system. From (2), (15) and (16), we have,

$$
\begin{aligned}
& \dot{V}(t)=\mathbf{x}(t)^{\mathrm{T}} \mathbf{P}_{1} \dot{\mathbf{x}}(t)+\dot{\mathbf{x}}(t)^{\mathrm{T}} \mathbf{P}_{1} \mathbf{x}(t) \\
& =\sum_{i=1}^{p} w_{i}\left[\begin{array}{c}
\mathbf{x}(t) \\
\mathbf{y}(t) \\
\mathbf{u}(t)
\end{array}\right]^{\mathrm{T}}\left(\mathbf{P}^{\mathrm{T}}\left[\begin{array}{ccc}
\mathbf{A}_{i} & \mathbf{0} & \mathbf{B}_{i} \\
\mathbf{0} & \mathbf{0} & \mathbf{0} \\
\mathbf{0} & \mathbf{0} & \mathbf{0}
\end{array}\right]+\left[\begin{array}{ccc}
\mathbf{A}_{i} & \mathbf{0} & \mathbf{B}_{i} \\
\mathbf{0} & \mathbf{0} & \mathbf{0} \\
\mathbf{0} & \mathbf{0} & \mathbf{0}
\end{array}\right]^{\mathrm{T}}\right)\left[\begin{array}{l}
\mathbf{x}(t) \\
\mathbf{y}(t) \\
\mathbf{u}(t)
\end{array}\right]
\end{aligned}
$$

where $\quad \mathbf{P}=\left[\begin{array}{ccc}\mathbf{P}_{1} & \mathbf{0} & \mathbf{0} \\ \mathbf{P}_{2} & \mathbf{P}_{3} & \mathbf{0} \\ \mathbf{P}_{4} & \mathbf{P}_{5} & \mathbf{P}_{6}\end{array}\right] \in \mathfrak{R}^{(2 n+m) \times(2 n+m)}, \quad \mathbf{P}_{2} \in \mathfrak{R}^{n \times n}, \quad \mathbf{P}_{3} \in \mathfrak{R}^{n \times n}$, $\mathbf{P}_{4} \in \Re^{m \times n}, \mathbf{P}_{5} \in \Re^{m \times n}, \mathbf{P}_{6} \in \mathfrak{R}^{m \times m}$. From (15) and (17), using the property of $\sum_{i=1}^{p} w_{i}=\sum_{j=1}^{p} m_{j}=\sum_{i=1}^{p} \sum_{j=1}^{p} w_{i} m_{j}=1$, we have, $\dot{V}(t)=\sum_{i=1}^{p} \sum_{j=1}^{p} w_{i} m_{j}\left[\begin{array}{l}\mathbf{x}(t) \\ \mathbf{y}(t) \\ \mathbf{u}(t)\end{array}\right]^{\mathrm{T}}\left(\mathbf{P}^{\mathrm{T}}\left[\begin{array}{ccc}\mathbf{A}_{i} & \mathbf{0} & \mathbf{B}_{i} \\ \mathbf{G}_{j} & -\mathbf{I} & \mathbf{0} \\ \mathbf{0} & \mathbf{F}_{j} & -\mathbf{I}\end{array}\right]+\left[\begin{array}{ccc}\mathbf{A}_{i} & \mathbf{0} & \mathbf{B}_{i} \\ \mathbf{G}_{j} & -\mathbf{I} & \mathbf{0} \\ \mathbf{0} & \mathbf{F}_{j} & -\mathbf{I}\end{array}\right]^{\mathrm{T}}\right)\left[\begin{array}{l}\mathbf{x}(t) \\ \mathbf{y}(t) \\ \mathbf{u}(t)\end{array}\right]$

Expanding (18), we have,

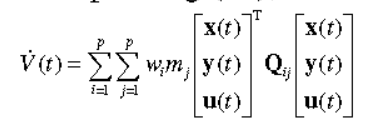

$\mathbf{Q}_{i j}=\left[\begin{array}{ccc}\mathbf{P}_{1}{ }^{\mathrm{T}} \mathbf{A}_{i}+\mathbf{A}_{i}{ }^{\mathrm{T}} \mathbf{P}_{1}+\mathbf{P}_{2}{ }^{\mathrm{T}} \mathbf{G}_{j}+\mathbf{G}_{j}{ }^{\mathrm{T}} \mathbf{P}_{2} & * & * \\ \mathbf{P}_{3}{ }^{\mathrm{T}} \mathbf{G}_{j}+\mathbf{F}_{j}{ }^{\mathrm{T}} \mathbf{P}_{4}-\mathbf{P}_{2} & \mathbf{F}_{j}{ }^{\mathrm{T}} \mathbf{P}_{5}+\mathbf{P}_{5}^{\mathrm{T}} \mathbf{F}_{j}-\mathbf{P}_{3}{ }^{\mathrm{T}}-\mathbf{P}_{3} & * \\ \mathbf{B}_{i}{ }^{\mathrm{T}} \mathbf{P}_{1}-\mathbf{P}_{4} & \mathbf{P}_{6}{ }^{\mathrm{T}} \mathbf{F}_{j}-\mathbf{P}_{5} & -\mathbf{P}_{6}{ }^{\mathrm{T}}-\mathbf{P}_{6}\end{array}\right], i, j$

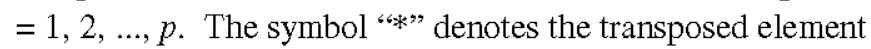
at the corresponding position. From (19), there are two cases to be investigated, namely, $m_{i}=w_{i}$ and $m_{i} \neq w_{i}$.

\section{A. Case 1: $m_{i}=w_{i}$ for all $i$}

Under such a condition, it is required that both the fuzzy plant model and the fuzzy controller share the same premise rules. As the grades of membership $\left(m_{i}\right)$ are required to be known in value, the fuzzy plant model needs to be uncertainty free. This condition simplifies the stability analysis and leads to relaxed stability conditions [4]-[12]. It can be seen from (19) that there is no crossed terms $i$ and $j$ in each element of $\mathbf{Q}_{i j}$. Hence, considering $m_{i}=w_{i}$ for all $i$ and using the property of $\sum_{i=1}^{p} w_{i}=\sum_{i=1}^{p} \sum_{j=1}^{p} w_{i} w_{j}=1$, we have,

$\dot{V}(t)=\sum_{i=1}^{p} \sum_{j=1}^{p} w_{i} w_{j}\left[\begin{array}{l}\mathbf{x}(t) \\ \mathbf{y}(t) \\ \mathbf{u}(t)\end{array}\right]^{\mathrm{T}} \mathbf{Q}_{i j}\left[\begin{array}{l}\mathbf{x}(t) \\ \mathbf{y}(t) \\ \mathbf{u}(t)\end{array}\right]=\sum_{i=1}^{p} w_{i}\left[\begin{array}{l}\mathbf{x}(t) \\ \mathbf{y}(t) \\ \mathbf{u}(t)\end{array}\right]^{\mathrm{T}} \mathbf{Q}_{i i}\left[\begin{array}{l}\mathbf{x}(t) \\ \mathbf{y}(t) \\ \mathbf{u}(t)\end{array}\right]$

It can be seen from $(20)$ that $\dot{V}(t)<0$ (which implies the asymptotic stability of the fuzzy-model-based control system) if $\mathbf{Q}_{i i}<0$ for all $i$. The stability analysis result is summarized in the following theorem.

Theorem 1: The fuzzy-model-based control system of (12), formed by the nonlinear plant in the form of (2) and the fuzzy controller of (11) connected in a closed loop, and the membership function of the fuzzy controller are designed as $m_{i}(\mathbf{x}(t))=w_{i}(\mathbf{x}(t))$ for all $i$, is guaranteed to be asymptotically stable if there exist matrices $\mathbf{P}_{1}=\mathbf{P}_{1}^{\mathrm{T}} \in \mathfrak{R}^{n \times n}$, $\mathbf{P}_{2} \in \mathfrak{R}^{n \times n}, \mathbf{P}_{3} \in \mathfrak{R}^{n \times n}, \mathbf{P}_{4} \in \mathfrak{R}^{m \times n}, \mathbf{P}_{5} \in \mathfrak{R}^{m \times n}$ and $\mathbf{P}_{6} \in \mathfrak{R}^{m \times m}$ such that the following LMIs are satisfied.
$\mathbf{P}_{1}>0 ; \mathbf{Q}_{i i}<0, i=1,2, \ldots, p$. The feedback gains of $\mathbf{F}_{i}$ and $\mathbf{G}_{i}, i=1,2, \ldots, p$, are determined prior to applying the theorem.

Remark 2: It can be seen from (13) and (14) that the fuzzy controller of (11) is decomposed into the form of (15). In (15), the double summation expression appearing in (11) is effectively reduced to a single summation expression. As a result, unlike the analysis approach in [3]-[12], the number of stability conditions and the computational complexity can be reduced as the stability conditions are not necessarily to be formulated as large matrices incorporating all linear sub-control systems. When the condition of $m_{i}=w_{i}$ and the feedback gains $\mathbf{G}_{i}=\mathbf{I}$ are considered, the number of stability conditions is $p(p+1)$ in [4]-[12]. Furthermore, the system stability is governed by a large matrix composed of all the linear sub-control systems. The large matrix in the stability conditions increases the computational demand, especially when the value of $p$ is large. When $\mathbf{G}_{i}$ is not equal to $\mathbf{I}$, the number of stability conditions will be further increased by using the stability analysis approach in [4]-[12]. In this paper, it can be seen from Theorem 1 that the number of stability conditions is reduced to $p$ (even for $\mathbf{G}_{i}$ being not equal to $\mathbf{I}$ ). The computational demand for solving the solution can be lowered.

\section{B. Case 2: $m_{i} \neq w_{i}$ for all $i$}

Under such a condition, as the fuzzy plant model and the fuzzy controller do not share the same premise rules, the grades of membership $\left(w_{i}\right)$ of the fuzzy plant model are not necessarily known. Hence the nonlinear plant to be controlled can have parameter uncertainties that are absorbed into the membership functions of the fuzzy plant model. The fuzzy controller with $m_{i} \neq w_{i}$ is thus relatively robust to the plant parameter uncertainties. Also, simple and commonly-used membership functions can be employed to realize the fuzzy controller. However, when the premise rules are different, the stability conditions will usually be very conservative.

In the following, to retain the advantages of the fuzzy controller with $m_{i} \neq w_{i}$, and reduce the conservativeness of the stability conditions, the membership functions of the fuzzy controller are designed with $m_{i}-\rho w_{i}>0$ for all $\mathbf{x}(t)$ and $i$, where $0<\rho<1$. Under such a design criterion, some free matrices can be added to ease the stability analysis and lead to less conservative stability conditions. From (19), considering $m_{i}-\rho w_{i}>0$ for all $\mathbf{x}(t)$ and $i$, and using the property of $\sum_{i=1}^{p} w_{i}=\sum_{j=1}^{p} m_{j}=\sum_{i=1}^{p} \sum_{j=1}^{p} w_{i} m_{j}=1$, we have, $\dot{V}(t)=\sum_{i=1}^{p} \sum_{j=1}^{p} w_{i} m_{i}\left[\begin{array}{l}\mathbf{x}(t) \\ \mathbf{y}(t) \\ \mathbf{u}(t)\end{array}\right]^{\mathrm{T}} \mathbf{Q}_{i j}\left[\begin{array}{l}\mathbf{x}(t) \\ \mathbf{y}(t) \\ \mathbf{u}(t)\end{array}\right]$

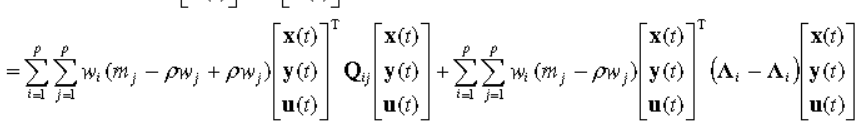
$=\rho \sum_{i=1}^{p} w_{i}\left[\begin{array}{l}\mathbf{x}(t) \\ \mathbf{y}(t) \\ \mathbf{u}(t)\end{array}\right]^{\mathrm{T}}\left(Q_{i}-\frac{1-\rho}{\rho} \Lambda_{i}\right)\left[\begin{array}{l}\mathbf{x}(t) \\ \mathbf{y}(t) \\ \mathbf{u}(t)\end{array}\right]+\sum_{i=1}^{p} \sum_{j=1}^{p} w_{i}\left(m_{j}-\rho w_{j}\right)\left[\begin{array}{l}\mathbf{x}(t) \\ \mathbf{y}(t) \\ \mathbf{u}(t)\end{array}\right]^{\mathrm{T}}\left(Q_{i j}+\Lambda_{i} ;\left[\begin{array}{l}\mathbf{x}(t) \\ \mathbf{y}(t) \\ \mathbf{u}(t)\end{array}\right]\right.$

where $\boldsymbol{\Lambda}_{i}=\boldsymbol{\Lambda}_{i}^{\mathrm{T}} \in \mathfrak{R}^{(2 n+m) \times(2 n+m)}, i=1,2, \ldots, p$, are arbitrary matrices which are introduced to compensate the unstable system elements to alleviate the conservativeness of the stability analysis. It can be seen from (21) that $\dot{V}(t)<0$ 
(which implies the asymptotic stability of the fuzzy-model-based control system) if $\mathbf{Q}_{i i}-\frac{1-\rho}{\rho} \boldsymbol{\Lambda}_{i}<0$ and $\mathbf{Q}_{i j}+\boldsymbol{\Lambda}_{i}<0$ for all $i$ and $j$. The stability analysis result is summarized in the following theorem.

Theorem 2: The fuzzy-model-based control system of (12), formed by the nonlinear plant in the form of (2) and the fuzzy controller of (11) connected in a closed loop, and the membership function of the fuzzy controller are designed as $m_{i}(\mathbf{x}(t))-\rho w_{i}(\mathbf{x}(t))>0$ for all $\mathbf{x}(t)$ and $i$, where $0<\rho<1$, is guaranteed to be asymptotically stable if there exist matrices $\mathbf{P}_{1}=\mathbf{P}_{1}^{\mathrm{T}} \in \mathfrak{R}^{n \times n}, \mathbf{P}_{2} \in \mathfrak{R}^{n \times n}, \mathbf{P}_{3} \in \mathfrak{R}^{r \times n}, \mathbf{P}_{4} \in \mathfrak{R}^{m \times n}$, $\mathbf{P}_{5} \in \mathfrak{R}^{m \times n}, \quad \mathbf{P}_{6} \in \mathfrak{R}^{m \times m}$ and $\boldsymbol{\Lambda}_{i}=\boldsymbol{\Lambda}_{i}{ }^{\mathrm{T}} \in \mathfrak{R}^{(2 n+m) \times(2 n+m)}$ such that the following LMIs are satisfied.

$\mathbf{P}_{1}>0 ; \mathbf{Q}_{i i}-\frac{1-\rho}{\rho} \boldsymbol{\Lambda}_{i}<0, i=1,2, \ldots, p ; \mathbf{Q}_{i j}+\boldsymbol{\Lambda}_{i}<0, i, j=$ $1,2, \ldots, p$. The feedback gains of $\mathbf{F}_{i}$ and $\mathbf{G}_{i}, i=1,2, \ldots, p$, are determined prior to applying the theorem.

Remark 3: The analysis approach given in this paper can be generalized to a fuzzy controller with the fuzzy feedback gains in the general form

$\mathbf{u}(t)=\sum_{i_{1}=1}^{p} \sum_{i_{2}=1}^{p} \cdots \sum_{i_{r}=1}^{p} m_{i_{1}} m_{i_{2}} \cdots m_{i_{r}} \mathbf{F}_{i_{1}} \mathbf{G}_{i_{2}} \cdots \mathbf{G}_{i_{r}} \mathbf{x}(t)$

where

$\mathbf{F}_{\dot{1}_{1}} \in \mathfrak{R}^{m \times n}$ and $\mathbf{G}_{i_{1}}, \mathbf{G}_{i_{2}}, \ldots, \mathbf{G}_{i_{r}} \in \mathfrak{R}^{m \times n}$ are feedback gains and $r$ is a non-zero positive integer. From (13)-(15), we can see that the proposed analysis approach will not increase the number of stability conditions. Although the dimension of the matrices in the stability conditions will increase, the feedback gains of $\mathbf{G}_{i_{1}}, \mathbf{G}_{i_{2}}, \ldots, \mathbf{G}_{i_{r}}$

can enhance the nonlinearity of the fuzzy controller to compensate the unstable elements of the nonlinear plant in order to produce less conservative stability conditions. When $\mathbf{G}_{i_{1}}=\mathbf{G}_{i_{2}}=\ldots=\mathbf{G}_{i_{r}}=\mathbf{I}$, where $\mathbf{I}$ is the identity matrices, the proposed fuzzy controller is reduced to the traditional one in [3]-[12].

In Theorem 1 and Theorem 2, the values the feedback gains of $\mathbf{F}_{i}$ and $\mathbf{G}_{i}$ have to be determined before finding the solution of the stability conditions. In the following, the feedback gains of $\mathbf{F}_{i}$ and $\mathbf{G}_{i}$ are represented as the decision variables of the LMI problem. Hence, their values can be obtained automatically by using some convex programming techniques.

Let $\quad \mathbf{X}_{1}=\mathbf{X}_{1}^{\mathrm{T}}=\mathbf{P}_{1}^{-1} \in \mathfrak{R}^{n \times n}>0$

$\mathbf{X}=\left[\begin{array}{ccc}\mathbf{X}_{1} & \mathbf{0} & 0 \\ \mathbf{X}_{2} & \mathbf{X}_{3} & 0 \\ \mathbf{X}_{4} & \mathbf{X}_{5} & \mathbf{X}_{6}\end{array}\right]=\mathbf{P}^{-1} \in \mathfrak{R}^{(2 n+m) \times(2 n+m)} \quad, \quad \mathbf{X}_{2} \in \mathfrak{R}^{n \times n}, \quad \mathbf{X}_{3} \in \mathfrak{R}^{r \times n}$, $\mathbf{X}_{4} \in \mathfrak{R}^{n \times n}, \mathbf{X}_{5} \in \mathfrak{R}^{n \times n}, \mathbf{X}_{6} \in \mathfrak{R}^{m \times m}$. To facilitate the formulation of the LMI stability conditions, the conservativeness will be increased. Let $\mathbf{X}_{2}=\mathbf{X}_{3} \in \Re^{r \times n}$, we design the feedback gains of $\mathbf{F}_{i}$ and $\mathbf{G}_{i}$ as $\mathbf{K}_{i} \mathbf{X}_{1}^{-1}$ and $\mathbf{N}_{i} \mathbf{X}_{2}^{-1}$ respectively, where $\mathbf{K}_{i} \in \mathfrak{R}^{n \times n}$ and $\mathbf{N}_{i} \in \mathfrak{R}^{n \times n}$. Pre- and post-multiplying $\mathbf{X}$ and $\mathbf{X}^{\mathrm{T}}$ to the stability conditions in Theorem 1 and Theorem 2, and letting
$\mathbf{V}_{i}=\mathbf{V}_{i}^{\mathrm{T}}=\mathbf{X} \mathbf{\Lambda}_{i} \mathbf{X}^{\mathrm{T}} \in \mathfrak{R}^{(2 n+m) \times(2 n+m)}$, we have the following theorems governing the system stability of the fuzzy-model-based control system of (12) with $w_{i}=m_{i}$ and $w_{i}$ $\neq m_{i}$ respectively:

Theorem 3: The fuzzy-model-based control system of (12), formed by the nonlinear plant in the form of (2), and the fuzzy controller of (11) connected in a closed loop and the membership function of the fuzzy controller are designed as $m_{i}(\mathbf{x}(t))=w_{i}(\mathbf{x}(t))$ for all $i$, is guaranteed to be asymptotically stable if there exist matrices $\mathbf{X}_{1}=\mathbf{X}_{1}{ }^{\mathrm{T}} \in \mathfrak{R}^{n \times n}$, $\mathbf{X}_{2} \in \mathfrak{R}^{n \times n}, \mathbf{X}_{4} \in \mathfrak{R}^{n \times n}, \mathbf{X}_{5} \in \mathfrak{R}^{n \times n}, \mathbf{X}_{6} \in \mathfrak{R}^{n \times m}, \mathbf{K}_{i} \in \mathfrak{R}^{r \times n}$ and $\mathbf{N}_{i} \in \mathfrak{R}^{m \times n}$ such that the following LMIs are satisfied.

$$
\mathbf{X}_{1}>0 ;\left[\begin{array}{ccc}
\mathbf{A}_{i} \mathbf{X}_{1}+\mathbf{X}_{1} \mathbf{A}_{i}{ }^{\mathrm{T}}+\mathbf{B}_{i} \mathbf{X}_{4}+\mathbf{X}_{4}{ }^{\mathrm{T}} \mathbf{B}_{i}{ }^{\mathrm{T}} & * & * \\
\mathbf{N}_{i}-\mathbf{X}_{2}+\mathbf{X}_{5}{ }^{\mathrm{T}} \mathbf{B}_{i}{ }^{\mathrm{T}} & -\mathbf{X}_{2}-\mathbf{X}_{2}{ }^{\mathrm{T}} & * \\
\mathbf{K}_{i}-\mathbf{X}_{4}+\mathbf{X}_{6}{ }^{\mathrm{T}} \mathbf{B}_{i}{ }^{\mathrm{T}} & \mathbf{K}_{i}-\mathbf{X}_{5} & -\mathbf{X}_{6}-\mathbf{X}_{6}{ }^{\mathrm{T}}
\end{array}\right]<0, i=1,
$$

$2, \ldots, p$, and the feedback gains of $\mathbf{F}_{i}$ and $\mathbf{G}_{i}, i=1,2, \ldots, p$, are designed as $\mathbf{K}_{i} \mathbf{X}_{1}^{-1}$ and $\mathbf{N}_{i} \mathbf{X}_{2}^{-1}$ respectively.

Theorem 4: The fuzzy-model-based control system of (12), formed by the nonlinear plant in the form of (2) and the fuzzy controller of (11) connected in closed loop, and the membership function of the fuzzy controller are designed as $m_{i}(\mathbf{x}(t))-\rho w_{i}(\mathbf{x}(t))>0$ for all $\mathbf{x}(t)$ and $i$, where $0<\rho<1$, is guaranteed to be asymptotically stable if there exist matrices $\quad \mathbf{X}_{1}=\mathbf{X}_{1}^{\mathrm{T}} \in \mathfrak{R}^{n \times n}, \quad \mathbf{X}_{2} \in \mathfrak{R}^{n \times n}, \quad \mathbf{X}_{4} \in \mathfrak{R}^{n \times n}$, $\mathbf{X}_{5} \in \mathfrak{R}^{n \times n}$ and $\mathbf{X}_{6} \in \mathfrak{R}^{n \times m}, \mathbf{K}_{i} \in \mathfrak{R}^{r \times n}, \mathbf{N}_{i} \in \mathfrak{R}^{\text {n×x }}$ and $\mathbf{V}_{i}=\mathbf{V}_{i}^{\mathrm{T}} \in \mathfrak{R}^{(2 n+m) \times(2 n+m)}$ such that the following LMIs are satisfied.

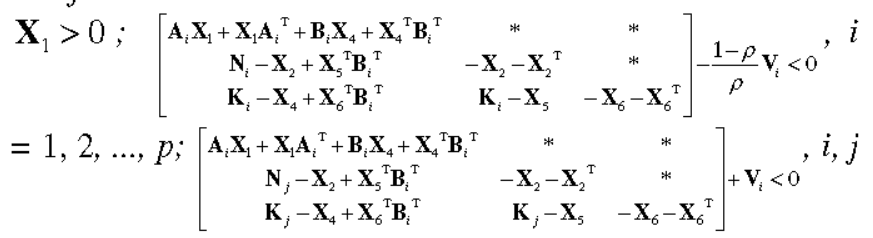
$=1,2, \ldots, p$, and the feedback gains of $\mathbf{F}_{i}$ and $\mathbf{G}_{i}, i=1,2, \ldots$, $p$, are designed as $\mathbf{K}_{i} \mathbf{X}_{1}^{-1}$ and $\mathbf{N}_{i} \mathbf{X}_{2}^{-1}$ respectively.

Remark 3: The stability conditions in Theorem 3 and Theorem 4 are valid if $\mathbf{X}^{-1}$ exists. It can be seen from Theorem 3 and Theorem 4 that if there exist a solution to the stability conditions, we have $\mathbf{X}_{1}>$ $0,-\mathbf{X}_{2}-\mathbf{X}_{2}{ }^{\mathrm{T}}<0$ and $-\mathbf{X}_{6}-\mathbf{X}_{6}{ }^{\mathrm{T}}<0$ (and $\mathbf{X}_{2}=\mathbf{X}_{3}$ ). These are the sufficient conditions to guarantee the existence of the inverse of $\mathbf{X}$.

\section{SimULATION EXAMPLES}

Two simulation examples are given in this section to illustrate the merits of the proposed approach.

\section{A. Simulation Example 1}

In this example, the stability regions of stability conditions in Theorem 1, Theorem 2 and the published stability conditions in [3]-[12] are compared. We consider the same fuzzy model given in [11] with the rules defined as follows. 
Rule $i$ : $\operatorname{IF} x_{1}(t)$ is $\mathrm{M}_{1}^{i}$

$$
\text { THEN } \dot{\mathbf{x}}(t)=\mathbf{A}_{i} \mathbf{x}(t)+\mathbf{B}_{i} u(t), i=1,2
$$

where $\mathbf{A}_{1}=\left[\begin{array}{cc}2 & -10 \\ 1 & 0\end{array}\right]$ and $\mathbf{A}_{2}=\left[\begin{array}{cc}a & -10 \\ 1 & 3\end{array}\right] ; \quad \mathbf{B}_{1}=\left[\begin{array}{l}1 \\ 0\end{array}\right]$ and $\mathbf{B}_{2}=\left[\begin{array}{l}b \\ 0\end{array}\right]$; $\mathbf{x}(t)=\left[\begin{array}{ll}x_{1}(t) & x_{2}(t)\end{array}\right]^{\mathrm{T}},-20 \leq a \leq 20$ and $1 \leq b \leq 70$. The traditional fuzzy controllers in the form of $u(t)=\sum_{j=1}^{2} m_{i} \mathbf{F}_{j} \mathbf{x}(t)$, where the membership functions $m_{i}=w_{i}[4]-[12]$ and $m_{i} \neq w_{i}$ [3]-[4] for all $i$, are employed to close the feedback loop. The feedback gains $\mathbf{F}_{j}$ are designed such that the eigenvalues of $\mathbf{A}_{i}+\mathbf{B}_{i} \mathbf{F}_{i}, i=1,2$, are located at -5 and -5 . Fig. 1 shows the stability regions given by the published stability conditions in [4]-[12] for $w_{i}=m_{i}$ and [3]-[4] for $w_{i} \neq m_{i}$.

The proposed stability conditions in Theorem 1 and Theorem 2 are employed to deal with the fuzzy system for comparison. By employing similar design criterion, the feedback gains $\mathbf{G}_{i}$ are designed arbitrarily such that the eigenvalues of $\mathbf{A}_{i}+\mathbf{B}_{i} \mathbf{F}_{i} \mathbf{G}_{i}, i=1,2$, are located at -5 and -5 . Fig. 2 shows the stability region for the stability conditions in Theorem 1 with $m_{i}=w_{i}$. It can been seen from Fig. 1 and Fig. 2 that the proposed stability conditions in Theorem 1 produces a much larger stability region than that of the stability conditions given in [4]-[12]. It is mainly due to the extra feedback gains $\mathbf{G}_{i}$ that offer a higher degree of freedom to enhance the nonlinearity of the fuzzy controller in order to compensate the unstable elements of the fuzzy plant model. Fig. 3a shows the stability region for the stability conditions in Theorem 2 with $\rho=0.9$ and $m_{i} \neq w_{i}$. It can be seen from Fig. $1 \mathrm{a}$ and Fig. $3 \mathrm{a}$ that the proposed stability conditions in Theorem 2 produces a much larger stability region than that for the stability conditions given in [3]-[4]. Apart from the extra feedback gains $\mathbf{G}_{i}$, under the case of $m_{i} \neq w_{i}$, the larger stability region is achieved mainly due to the fact that the membership functions of both the fuzzy plant model and the fuzzy are considered during the stability analysis. Fig. $3 b$ shows the stability region for the stability conditions in Theorem 2 with $\rho=0.8$. It can be seen that a larger value of $\rho$ offers a larger stability region as the membership functions of the fuzzy plant model and fuzzy controller are closer to each other. Comparing with the stability conditions in Theorem 2 with $m_{i} \neq w_{i}$, the stability conditions in Theorem 1 with $m_{i}=$ $w_{i}$ will usually offer a larger stability region.

\section{B. Simulation Example 2}

In this simulation example, we illustrate that the proposed fuzzy controller has a stronger stabilization ability to deal with nonlinear systems as compared with the traditional fuzzy controller in [3]-[12]. Considering the nonlinear system described by a fuzzy model with the rules of (22), the membership functions are taken as $w_{1}\left(x_{1}(t)\right)=\mu_{\mathrm{M}_{1}^{1}}\left(x_{1}(t)\right)=$ $\left(1-\frac{c(t) \sin \left(\left.x_{1}(t)\right|^{-4}\right)^{5}}{1+e^{-100 x_{1}(t)^{3}\left(1-x_{1}(t)\right)}}\right) \frac{\cos \left(x_{1}(t)\right)^{2}}{1+e^{-2 s_{1}(t)(s)\left(3+x_{1}(t)\right)(0.42)}}$ and $w_{2}\left(x_{1}(t)\right)=\mu_{\mathrm{M}_{1}^{2}}\left(x_{1}(t)\right)=$ $1-\mu_{\mathrm{M}_{1}^{1}}\left(x_{1}(t)\right)$.
The proposed fuzzy controller with two rules is employed to deal with the nonlinear plant represented by the fuzzy model. When the case of $m_{i}=w_{i}$ for all $i$ is considered, based on the same design criterion of the fuzzy controller in the previous example, the values of the feedback gains are obtained as $\mathbf{F}_{1}=\left[\begin{array}{ll}-11.9999 & -14.9995\end{array}\right], \mathbf{F}_{2}=[-0.0448$ $-0.8060] ; \mathbf{G}_{1}=\left[\begin{array}{ll}0.3903 & 0.4878 \\ 0.4878 & 0.6098\end{array}\right], \mathbf{G}_{2}=\left[\begin{array}{ll}0.0031 & 0.0554 \\ 0.0554 & 0.9969\end{array}\right]$ for $a=-10, b$ $=67$ and $c(t)=0.1$. Fig. 4 shows the system state responses on using the traditional and the proposed fuzzy controllers under the initial system states of $\mathbf{x}(0)=\left[\begin{array}{ll}1 & 0.25\end{array}\right]$. Referring to this figure, it can be seen that the proposed fuzzy controller is able to stabilize the nonlinear plant (solid line) while the traditional one cannot (dotted line). The membership functions of the fuzzy model are highly nonlinear in nature and very complicated for implementation. As a result, the fuzzy controller employing the same membership functions as those of the fuzzy model will be very complicated in structure. To lower the implementation cost, the fuzzy controller can be realized by employing simple membership functions. Referring to the stability region shown in Fig. 3, which is under the case of $m_{i} \neq w_{i}$ and with $\rho=0.8$, it can be seen that the nonlinear plant with the proposed fuzzy controller is asymptotically stable for $a=-10$ and $b=33$. The membership functions of the fuzzy controller are designed as $m_{1}\left(x_{1}(t)\right)=$ $\mu_{\mathrm{N}_{1}^{1}}\left(x_{1}(t)\right)=0.725 e^{\frac{-\left(x_{1}(t)-0.38\right)^{2}}{2 \times 0.38^{2}}}+0.05$ and $m_{2}\left(x_{1}(t)\right)=$ $\mu_{\mathrm{N}_{1}^{2}}\left(x_{1}(t)\right)=1-\mu_{\mathrm{N}_{1}^{1}}\left(x_{1}(t)\right)$, which are simpler than those of the fuzzy model. It can be shown that the design condition of $m_{i}(\mathbf{x}(t))-\rho w_{i}(\mathbf{x}(t))>0$ holds for all $\mathbf{x}(t)$ and $i$, where $\rho=$ 0.8 and $c(t)=\frac{\left(\sin \left(x_{1}(t)\right)+1\right)}{20}$ (for the membership functions of the fuzzy plant model). In this example, $c(t)$ is regarded as the parameter uncertainty and is assumed to be uncertain in value for simulation. By finding the solution to the stability conditions in Theorem 2, we have $\mathbf{F}_{1}=\left[\begin{array}{lll}-11.9999 & -14.9995\end{array}\right]$, $\mathbf{F}_{2}=\left[\begin{array}{ll}-0.0909 & -1.6364\end{array}\right] ; \quad \mathbf{G}_{1}=\left[\begin{array}{ll}0.3903 & 0.4878 \\ 0.4878 & 0.6098\end{array}\right]$, $\mathbf{G}_{2}=\left[\begin{array}{ll}0.0031 & 0.0554 \\ 0.0554 & 0.9969\end{array}\right]$. Fig. 5 shows the system state responses (in solid lines) of the fuzzy-model-based control system. It can be seen that the nonlinear plant subject to parameter uncertainty can be successfully stabilized. Under the case of $m_{i} \neq w_{i}$, as the values of $w_{i}$ for all $i$ are not necessarily known, the fuzzy controller exhibits an inherent robustness property to deal with the nonlinear plant subject to parameter uncertainties. Furthermore, the stability conditions in [4]-[12] for the traditional fuzzy controller cannot be applied as the fuzzy model and fuzzy controller do not share the same membership function. For comparison purpose, the traditional fuzzy controller, with the feedback gains designed based on the criteria in Simulation Example 1, is employed. It can be seen from Fig. 5 that the traditional fuzzy controller (in dotted line) cannot stabilize the nonlinear plant.

To show the effect of the uncertainties of membership functions to the fuzzy-model-based control systems with the 
traditional fuzzy controller, we consider $a=-10$ and $b=9$. It can be checked with Fig. 2 and Fig. 3 that the traditional fuzzy controller can provide feasible solution for $m_{i}=w_{i}$ but not for $m_{i} \neq w_{i}$. Fig. 6 shows the system responses under these two cases. It can be seen that the traditional fuzzy controller with $m_{i}=w_{i}$ can stabilize the nonlinear plant but not $m_{i} \neq w_{i}$.

\section{CONCLUSION}

The stability of fuzzy-model-based control systems has been studied. A fuzzy controller with fuzzy feedback gains has been proposed. As a result, the proposed fuzzy controller exhibits stronger stabilization ability than a traditional fuzzy controller. To reduce the computational demand given by the increased order of the summation terms, the fuzzy controller has been separated from the overall system in formulating the system's dynamic model. Furthermore, under such a system representation, the number of stability conditions has been reduced. Two cases have been considered for various applications. LMI-based stability conditions have been derived using the Lyapunov-based approach. Simulation examples have been given to illustrate the effectiveness of the proposed approach.

\section{REFERENCES}

[1] T. Takagi and M. Sugeno, "Fuzzy identification of systems and its applications to modeling and control," IEEE Trans. Sys., Man., Cybern., vol. smc-15 no. 1, pp. 116-132, Jan. 1985.

[2] M. Sugeno and G.T. Kang, "Structure identification of fuzzy model," Fuzzy Sets and Systems, vol. 28, pp. 15-33, 1988.

[3] K. Tanaka and M. Sugeno, "Stability analysis and design of fuzzy control systems," Fuzzy Sets and Systems, vol. 45, pp.135-156, 1992.

[4] C.L. Chen, P.C. Chen, C.K. Chen, "Analysis and design of fuzzy control system," Fuzzy Sets and Systems, vol. 57, no 2, 26, pp. 125-140, Jul. 1993.

[5] S. Boyd, L. El Ghaoui, E. Feron, and V. Balakrishnan, Linear Matrix Inequalities in Systems and Control Theory, ser. SIAM studies in Applied Mathematics, Philadelphia, PA: SIAM, 1994.

[6] H.O. Wang, K. Tanaka, and M.F. Griffin, "An approach to fuzzy control of nonlinear systems: stability and the design issues," IEEE Trans. Fuzzy Syst., vol. 4, no. 1, pp. 14-23, Feb. 1996.

[7] K. Tanaka, T. Ikeda and H.O. Wang, "Fuzzy regulator and fuzzy observer: Relaxed stability conditions and LMI-based designs," IEEE Trans. Fuzzy Syst., vol. 6, no. 2, pp. 250-265, 1998.

[8] E. Kim and H. Lee, "New approaches to relaxed quadratic stability conditions of fuzzy control systems," IEEE Trans. Fuzzy Syst., vol. 8, no. 5 , pp. 523-534, 2000.

[9] X. Liu and Q. Zhang, "New approaches to $H_{\infty}$ controller designs based on fuzzy observers for T-S fuzzy systems via LMI," Automatica, vol. 39 , no. 9, pp. 1571-1582, Sep. 2003.

[10] X. Liu and Q. Zhang, "Approaches to quadratic stability conditions and $H_{\infty}$ control designs for T-S fuzzy systems," IEEE Trans. Fuzzy Syst., vol. 11, no. 6, pp. 830-839, 2003.

[11] M.C.M. Teixeira, E. Assunção, and R.G. Avellar, "On relaxed LMI-based designs for fuzzy regulators and fuzzy observers," IEEE Trans. Fuzzy Syst., vol. 11, no. 5, pp. 613-623, Oct. 2003.

[12] C.H. Fang, Y.S. Liu, S.W. Kau, L. Hong, and C.H. Lee, "A new LMI-based approach to relaxed quadratic stabilization of T-S fuzzy control systems," IEEE Trans. Fuzzy Syst., vol. 14, no. 3, pp. 386-397, Jun. 2006.

[13] T.M. Guerra and L. Vermeiren, "LMI-based relaxed nonquadratic stabilization conditions for nonlinear systems in the Takagi-Sugeno's form," Automatica, vol. 40, pp. 823-829, 2004.

[14] B.C. Ding, H.X. Sun, and P. Yang, "Further study on LMI-based relaxed nonquadratic stabilization conditions for nonlinear systems in the Takagi-Sugeno's form," Automatica, vol. 42, pp. 503-508, 2006.
[15] H.K. Lam and F.H.F. Leung, "Stability analysis and synthesis of fuzzy control systems subject to uncertain grades of membership," IEEE Trans. Syst., Man and Cybern., Part B: Cybernetics, vol. 35, no. 6, pp. 1322-1325, Dec. 2005.

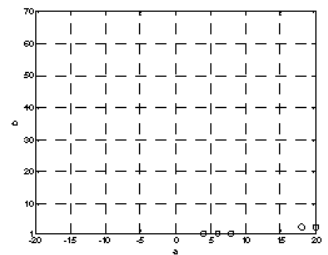

Fig. 1(a).

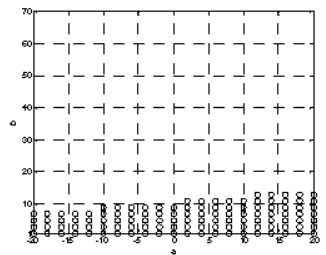

Fig. 1(b).
Fig. 1. Stability region for stability conditions in (a) [3]-[4] and (b) [4]-[12]

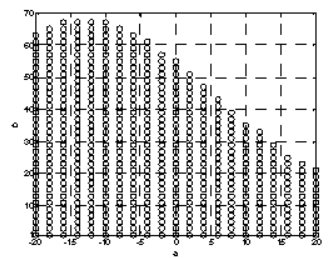

Fig. 2. Stability region for stability conditions in Theorem 1.

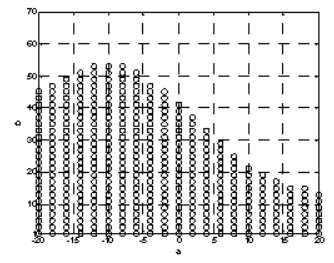

Fig. 3(a)

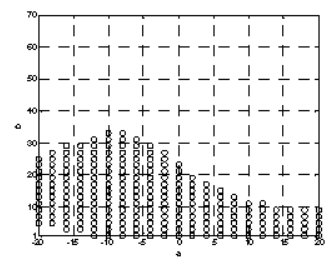

Fig. 3(b).
Fig. 3. Stability region for stability conditions in Theorem 3 with (a) $\rho=0.9$ and (b) $\rho=0.8$

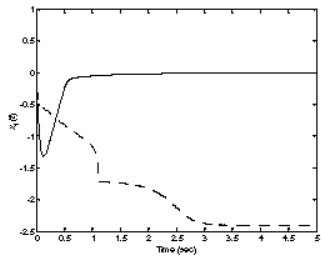

Fig. 4(a). $x_{1}(t)$.

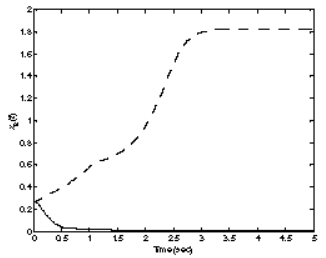

Fig. 4(b). $x_{2}(t)$.
Fig. 4. System states responses of the fuzzy-model-based control system under the case of $m_{i}=w_{i}$.

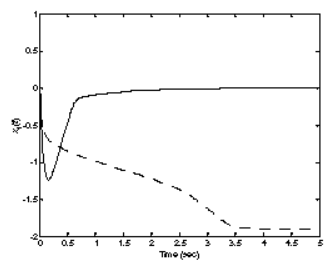

Fig. 5(a). $x_{1}(t)$

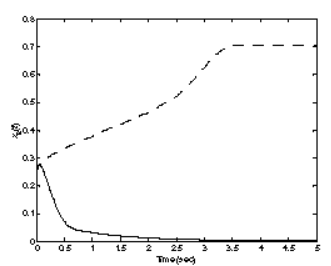

Fig. 5(b). $x_{2}(t)$.
Fig. 5. System states responses of the fuzzy-model-based control system under the case of $m_{i} \neq w_{i}$ and with $\rho=0.8$.

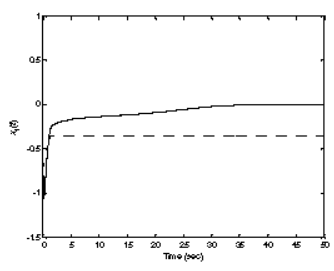

Fig. 6(a). $x_{1}(t)$.

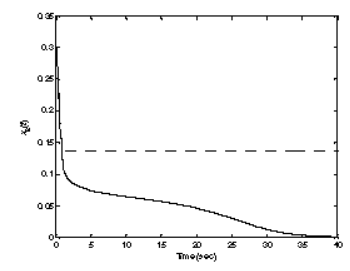

Fig. 6(b). $x_{2}(t)$
Fig. 6. System states responses of the fuzzy-model-based control system with traditional fuzzy controller under the cases of $m_{i}=w_{i}$ (dotted lines) and $m_{i} \neq$ $w_{i}$ (solid lines). 